\title{
TEMPORAL AND SPATIAL MRI RESPONSES TO SUBSECOND VISUAL ACTIVATION
}

\author{
P. Fransson, G. KrÜGer, K.D. Merboldt, and J. Frahm \\ Biomedizinische NMR Forschungs GmbH am Max-Planck-Institut für biophysikalische Chemie, \\ D-37070 Göttingen, Germany
}

\begin{abstract}
The temporal and spatial characteristics of oxygenation-sensitive MRI responses to very brief visual stimuli (five Hz reversing black and white checkerboard pattern versus darkness) were investigated (nine subjects) by means of serial single-shot gradient-echo echo-planar imaging $\left(2.0 \mathrm{~T}\right.$, TR $=400 \mathrm{~ms}$, mean $\mathrm{TE}=54 \mathrm{~ms}$, flip angle $\left.30^{\circ}\right)$. The use of a 0.2-s stimulus and a 90-s control phase resulted in an initial latency phase (about $2 \mathrm{~s}$, no signal change), a positive MRI response (2.5\% signal increase peaking at $5 \mathrm{~s}$ after stimulus onset), and a post-stimulus undershoot (1\% signal decrease peaking at $15 \mathrm{~s}$ after stimulus onset) lasting for about 50-60 s. The finding that a subsecond visual stimulus elicits both a strong positive MRI response and a long-lasting undershoot provides further evidence for the neuronal origin of slow signal fluctuations seen in the absence of functional challenge and their utility for mapping functional connectivity. The additional observation that a reduction of the inter-stimulus control phase from $90 \mathrm{~s}$ to $9.8 \mathrm{~s}$ does not seem to affect the spatial extent of cortical activation in pertinent maps is of major relevance for the design and analysis of "event-related" MRI studies. () 1998 Elsevier Science Inc.
\end{abstract}

Keywords: Neuroimaging; Human brain; Visual cortex; Functional activation; Brain function; Cerebral blood oxygenation.

\section{INTRODUCTION}

A current trend in magnetic resonance functional neuroimaging is the design of paradigms that improve the temporal efficiency of mapping studies and, perhaps more importantly, extend their applicability to established test protocols in cognitive neuropsychology. While exploiting the existing technical abilities for rapid data acquisition, pertinent attempts are based on the observation that even a subsecond visual stimulus may elicit a detectable magnetic resonance imaging (MRI) signal increase. ${ }^{1}$ A common strategy therefore follows the idea of rapidly presenting stimuli in a repetitive activation protocol. A typical example for functional mapping of "single events" 2 is the odd-ball paradigm designed to assess the brain's capability for detecting a novel event in a stream of rapidly presented stimuli. ${ }^{3}$ Accordingly, recent work addresses methodologic aspects that range from averaging MRI responses to "sin- gle trials" $", 5$ to deducing "transient responses" lyzing "event-related" data sets with use of linear ${ }^{7,8}$ or nonlinear models. ${ }^{9}$

A prerequisite for the development of valid approaches and a key issue in interpreting cerebral response characteristics is an understanding of the physiologic events that are associated with a change in neuronal activity and their modulation of oxygenationsensitive MRI signals. Specifically, the temporal evolution of the blood oxygenation level dependent (BOLD) MRI signal reflects changes in the net cerebral blood oxygenation via changes in the absolute concentration of paramagnetic deoxyhemoglobin within each image voxel. This level emerges as the result of multiple regulative mechanisms such as hemodynamic adjustments in cerebral blood volume and flow together with changes in oxidative metabolism. Because these processes inherently have different time constants, their relative contributions may critically affect the resulting response func-
RECEIVED 5/1/98; ACCEPTED 8/7/98.

P. Fransson is on leave from MR Research Center, Dept. of Clinical Neuroscience, Karolinska Institutet, Stockholm, Sweden.
Address correspondence to Dr. J. Frahm, Max-PlanckInstitut für biophysikalische Chemie, Biomedizinische NMR Forschungs GmbH, D-37070 Göttingen, Germany. E-mail: jfrahm@gwdg.de 
tion in relation to the actual timing of a repetitive protocol. This particularly holds true for paradigms that rely on rapid switches between different states of neural processing or employ short inter-stimulus (control) periods.

The model assumption of a standardized physiologic response function may be further complicated by differences in the vascular responsiveness of individual subjects caused by differences in age, medication, and (asymptomatic) cerebrovascular disease. Moreover, it cannot yet be excluded that physiologic responses reveal a stimulus or task dependence as recently observed for sustained visual activation ${ }^{10,11}$ or show a regional heterogeneity $^{12}$ within and between different brain systems due to differences in anatomy, vascularization, and neurotransmitter density.

Previous studies of very brief visual stimuli are controversial with regard to the post-stimulus undershoot phenomenon. A complete absence was reported by Savoy et al. ${ }^{1}$ employing stimulus durations of $1.0 \mathrm{~s}, 0.1 \mathrm{~s}$, and $17 \mathrm{~ms}$ (circular checkerboard) as well as by Konishi et al. ${ }^{6}$ for 2.0 -s and 0.2 -s stimuli $(10 \mathrm{~Hz}$ circular checkerboard), whereas a greatly diminished undershoot was found by $\mathrm{Hu}$ et al. ${ }^{13}$ for a 1.5 -s stimulus $(8.6 \mathrm{~Hz}$ LED goggles). On the other hand, functional spectroscopy revealed a 45-60 s undershoot for a 2.0-s stimulus ${ }^{14}$ (LED goggles) and in agreement with most published work using longer stimuli, we recently reported a marked post-stimulus undershoot (mean signal decrease of about $2 \%$ ) in response to a 1.6-s visual stimulus (10 Hz circular checkerboard). The signal required a recovery period of $60-90 \mathrm{~s}$ to fully return to pre-stimulation baseline. ${ }^{15}$

There is no doubt that details of the MRI response to brief visual stimuli are of utmost importance for the development of event-related magnetic resonance functional neuroimaging. For example, the occurrence of only a brief positive BOLD effect and/or the absence of an undershoot would allow responses to rapidly repeated stimuli to be treated as independent events. On the other hand, the prevalence of long-term effects would require much longer recovery periods to physiologically "decouple" cerebral responses and related MRI signal contributions. Therefore, the primary aim of this paper is to clarify the temporal and spatial characteristics of MRI responses to subsecond visual stimuli. Assessments of true changes in cerebral blood oxygenation were accomplished by gradient-echo EPI with BOLD MRI contrast.

\section{MATERIALS AND METHODS}

\section{Subjects and Functional Neuroimaging}

A total of 15 healthy volunteers participated in the study although the data from 6 subjects were discarded because of a lack of attention during the examination or

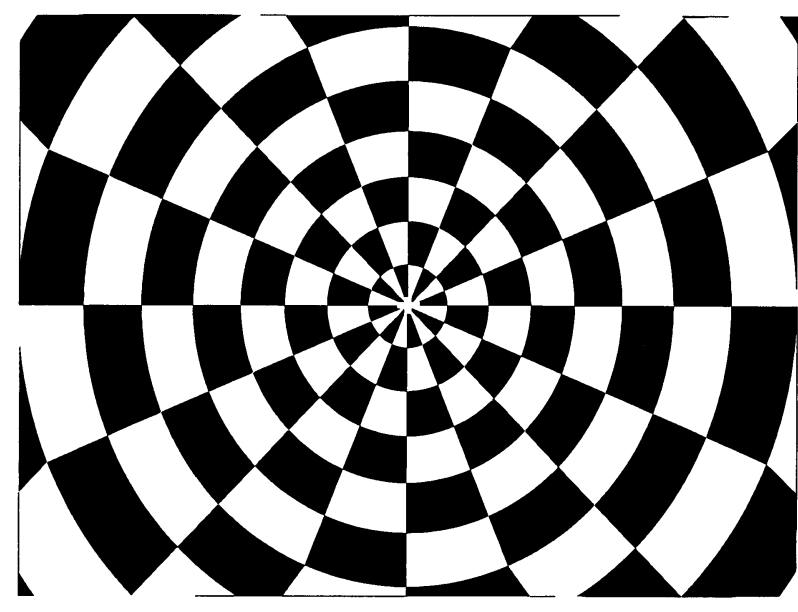

Fig. 1. The checkerboard stimulus represents a radial arrangement of 16 wedges formed by $7-8$ black and white segments at equal radial distance.

corruption/contamination of the image series by motion artifacts. The latter were detected by careful inspection of signal intensity time courses from high-contrast borders at the surface of the brain. The remaining nine subjects (age 24-33 years, mean 27.8 years) yielded a total of at least 6 successful experiments per activation protocol. Written informed consent was obtained from all subjects prior to the examinations.

All studies were conducted at 2.0 T (Siemens Vision, Erlangen, Germany) using the standard imaging headcoil. Dynamic oxygenation-sensitive MRI was performed by means of a single-shot, gradient-echo EPI sequence (mean TE $=54 \mathrm{~ms}$, symmetrical coverage of $\mathrm{k}$ space) using $\mathrm{T}_{2}{ }^{*}$-sensitivity in the absence of any $\mathrm{T}_{1}$ weighting, i.e., repetition times and flip angles were either $6.0 \mathrm{~s}$ and $90^{\circ}$ or $0.4 \mathrm{~s}$ and $30^{\circ}$, respectively. A single oblique section $(128 \times 128$ matrix, $384 \times 384$ $\mathrm{mm}$ field-of-view, 4-mm section thickness) along the calcarine fissure was selected to monitor functional responses in the visual cortex.

Visual activation employed a projection setup covering $40^{\circ} \times 30^{\circ}$ of the subject's visual field (Schäfter \& Kirchhoff, Hamburg, Germany). The checkerboard stimulus is shown in Fig. 1. Segment color was reversed 10 times per second, i.e., the repetition frequency of a full cycle was $5 \mathrm{~Hz}$ or required $0.2 \mathrm{~s}$. A red cross in the center of the checkerboard served as a fixation point. The control state was darkness. Subjects were instructed to keep their eyes open and maintain constant attention throughout the experiments.

\section{Activation Protocols}

Three activation protocols with identical stimuli but different timings were tested. The first protocol consisted 
of 6 repetitive cycles with $18 \mathrm{~s}$ stimulation and $36 \mathrm{~s}$ darkness (temporal resolution $6.0 \mathrm{~s}$, total measuring time $6 \mathrm{~min} 24 \mathrm{~s}$ ) to facilitate comparisons with previous work in this laboratory and to assess the subject's overall performance. Subsequent measurements addressed the responses to brief visual stimuli at a temporal resolution of $0.4 \mathrm{~s}$. In particular, the second protocol comprised four repetitive cycles with a stimulus duration of $0.2 \mathrm{~s}$ and a control phase of $90 \mathrm{~s}$. The third protocol kept the stimulus duration at $0.2 \mathrm{~s}$ but reduced the control phase to $9.8 \mathrm{~s}$ and increased the number of cycles to 12 .

Because the pre-stimulation baseline may differ from the inter-stimulus signal strength, its true value was determined during an initial control phase of $60 \mathrm{~s}$ preceding both protocols. Moreover, a final recovery period of $100 \mathrm{~s}$ after the last stimulation provided sufficient time to monitor the return to pre-stimulation conditions. The resulting total measuring time was $8 \min 41 \mathrm{~s}$ for the 0.2 $\mathrm{s} / 90 \mathrm{~s}$ protocol and $4 \mathrm{~min} 40 \mathrm{~s}$ for the $0.2 \mathrm{~s} / 9.8 \mathrm{~s}$ protocol, respectively. The first $8 \mathrm{~s}$ (20 images) of each protocol were discarded to account for equilibration purposes.

\section{Data Analysis}

Functional mapping was based on a cross-correlation analysis ${ }^{16}$ of individual pixel intensity time courses with a reference function representing the activation protocol. The actual waveform consisted of a boxcar function with amplitudes of 0 (darkness: baseline, control periods) and 1 (stimulation) and was shifted by $4.0 \mathrm{~s}$ with respect to stimulus onset to account for hemodynamic latencies. Quantitative maps of correlation coefficients were obtained by a statistical analysis that - based on the individual noise distribution - utilizes a high threshold for identifying activated centers $(p \leq 0.0001)$ and integrates neighboring pixels for area delineation. Details of the procedure have been published elsewhere. ${ }^{17}$ Color-coded maps were superimposed onto $\mathrm{T}_{1}$-weighted FLASH images depicting the anatomy and macrovasculature. Signal intensity time courses from dynamic EPI were obtained from all activated pixels admitted by the aforementioned analysis. MRI signal intensities were normalized to prestimulation baseline, i.e., the average signal strength observed prior to the first stimulation period in each experiment.

\section{RESULTS AND DISCUSSION}

Figure 2 presents typical activation maps indicating the spatial extent of visual processing elicited by the various protocols. In general, activation by the $18 \mathrm{~s} / 36 \mathrm{~s}$ protocol (Fig. 2b) resulted in a much larger area than obtained by either the $0.2 \mathrm{~s} / 90 \mathrm{~s}$ (Fig. 2c) or the $0.2 \mathrm{~s} / 9.8$ $\mathrm{s}$ protocol (Fig. 2d) and typically included not only primary visual cortices but also extrastriate areas. When averaged across subjects, the mean activated areas \pm standard deviation covered $165 \pm 47$ pixels $\left(1485 \mathrm{~mm}^{2}\right)$ for the $18 \mathrm{~s} / 36 \mathrm{~s}$ protocol, $47 \pm 14$ pixels $\left(423 \mathrm{~mm}^{2}\right)$ for the $0.2 \mathrm{~s} / 90 \mathrm{~s}$ protocol and $70 \pm 16$ pixels $\left(630 \mathrm{~mm}^{2}\right)$ for the $0.2 \mathrm{~s} / 9.8 \mathrm{~s}$ protocol. It is important to note that activated areas in response to both $0.2 \mathrm{~s}$ protocols largely overlap and at least qualitatively demonstrate spatial congruence independent of the used recovery period (90 $\mathrm{s}$ as opposed to $9.8 \mathrm{~s}$ ). The residual difference in the quantitative values must be ascribed to a sensitivity difference due to the actual number of cycles used (4 as opposed to 12) which also precludes a more detailed comparison of pertinent activation maps across all subjects.

The more extended activation by the much longer $18 \mathrm{~s}$ stimulus (Fig. 2b) may be best explained by differences in perceived stimulus quality. Although a slightly better contrast-to-noise may have contributed to a more robust data analysis, the more intriguing argument stems from the fact that the $18 \mathrm{~s}$ stimulus allows the subjects to experience all aspects of the $5 \mathrm{~Hz}$ reversing checkerboard paradigm, whereas the $0.2 \mathrm{~s}$ stimulus merely represents a single reversal of black and white segments. It is sufficient to consciously experience the checkerboard pattern, but too short for a comprehension of movement, so that pertinent bilateral activations in extrastriate cortex are missing in the $0.2 \mathrm{~s}$ maps (Fig. 2c,d).

\section{The 0.2 s/90 s Protocol}

A set of mean time courses of normalized MRI signal intensity changes is shown in Fig. 3 for the $0.2 \mathrm{~s} / 90 \mathrm{~s}$ protocol. The actual curves refer to the real-time data (Fig. 3a) and the time-locked average of all 4 activation cycles covering either the full cycle (Fig. 3b) or only the first $10 \mathrm{~s}$ (Fig. 3c). The resulting response profile is characterized by an initial latency phase of about $2.0 \mathrm{~s}$ followed by a $2.5 \%$ signal increase peaking at about $5.0 \mathrm{~s}$ after stimulation onset. The positive BOLD response is followed by an undershoot with signal intensities decreasing by about $1 \%$ ( $15 \mathrm{~s}$ after stimulus onset) below the pre-stimulation baseline. Return to the pre-stimulation baseline is accomplished only after a $50-60 \mathrm{~s}$ recovery period.

The data unambiguously demonstrate that a 0.2 visual stimulus is sufficient to elicit both an $8 \mathrm{~s}$ positive BOLD effect and an even longer post-stimulus undershoot, i.e., a physiologic sequence of hemodynamic and metabolic adjustments with a total duration of about one minute. It is noteworthy that the overall response profile including the absence of a signal decrease or "dip" during the initial latency phase is in close agreement with previous results obtained for an identical though eightfold longer 1.6 s stimulus. ${ }^{15}$ Hence, it seems impossible to avoid a temporal overlap of individual MRI-detected BOLD- 

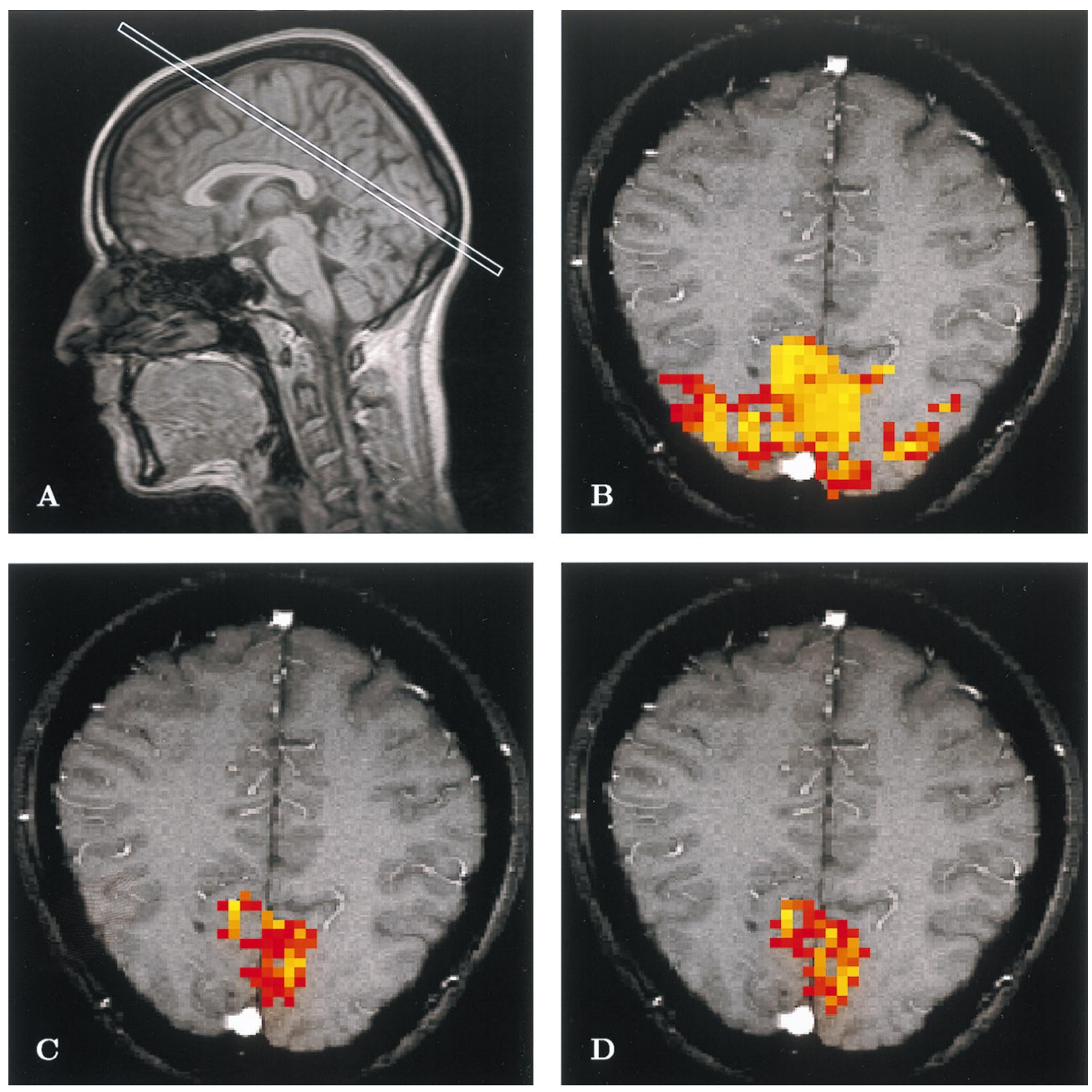

Fig. 2. A, section orientation for a temporal and spatial analysis of functional responses to visual activation in human occipital cortex (B-D). Color-coded activation maps of one subject superimposed onto an anatomic reference image (rf-spoiled FLASH, TR/TE $=$ $70 / 6 \mathrm{~ms}$, flip angle $60^{\circ}$ ) delineating the spatial extent of responses to a 18 -s/36-s protocol (B), a $0.2-\mathrm{s} / 90$-s protocol (C), and a 0.2 -s/9.8-s (D) protocol (5 Hz reversing black and white checkerboard versus darkness).

type responses to rapidly presented stimuli by shortening their duration. Any event-related paradigm design has to take this problem into account.

\section{The 0.2 s/9.8 s Protocol}

Figure 4 compares the real-time MRI signal intensity changes for the $0.2 \mathrm{~s} / 9.8 \mathrm{~s}$ protocol (Fig. 4a) to the time-locked average of all 12 (Fig. 4b) or only the first 4 activation cycles (Fig. 4c). For this rapid repetitive protocol only the real-time data allow an adequate analysis of the temporal evolution of signal changes as the individual activation cycles result in MRI responses that are no longer equivalent. Instead, because insufficient recovery periods between stimuli preclude a full recovery to pre-stimulation conditions, the MRI signal reaches a new equilibrium after 3-4 stimulations with a relative mean signal strength of about $1 \%$ below the pre-stimulation baseline. Thus, the inter-stimulus signal strength reflects the temporal overlap of physiologic responses to successive stimuli, e.g., of positive BOLD responses and preceding undershoot phenomena. For the timing parameters chosen here, the "functional contrast" between stimulation and control, i.e., the peak-to-peak signal difference, remains almost unchanged throughout the experiment at about $2.5 \%$.

In close analogy to the data shown in Fig. 3, the final end of stimulation is characterized by a slow return to pre-stimulation baseline requiring about 50-60 s for full recovery. When calculating a time-locked average of all 12 activation cycles (Fig. 4b) the apparent inter-stimulus "baseline" during steady-state conditions turns out to be much lower than the true pre-stimulation baseline. Av- 


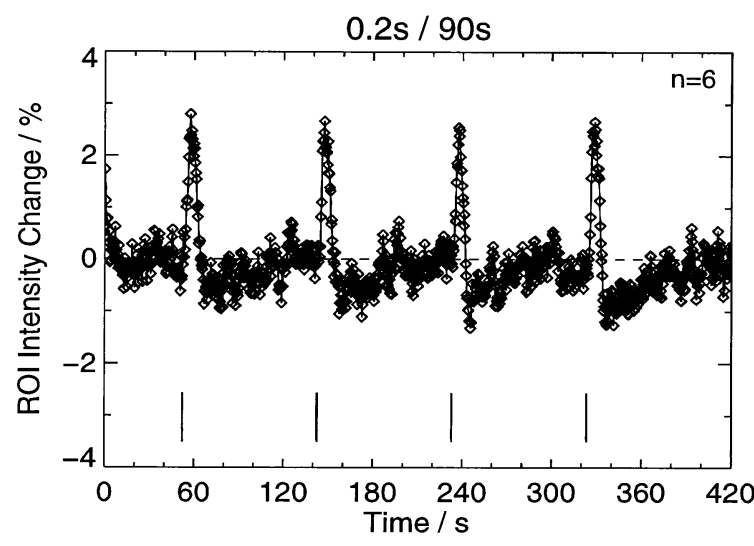

(A)

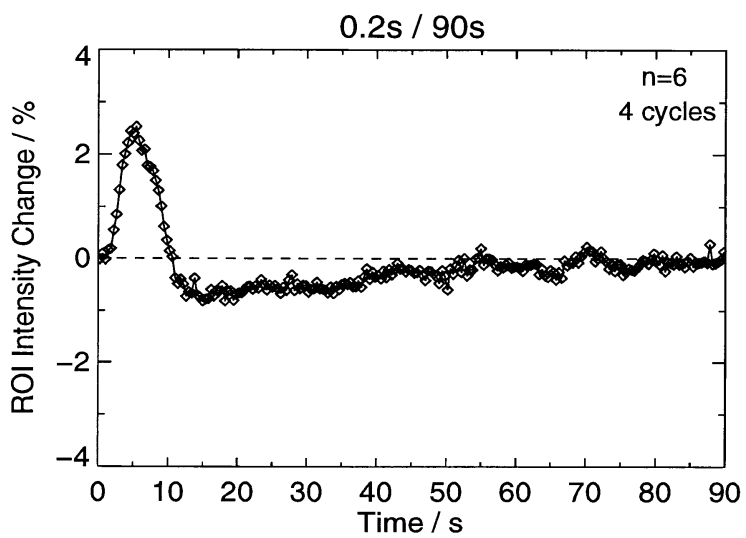

(B)

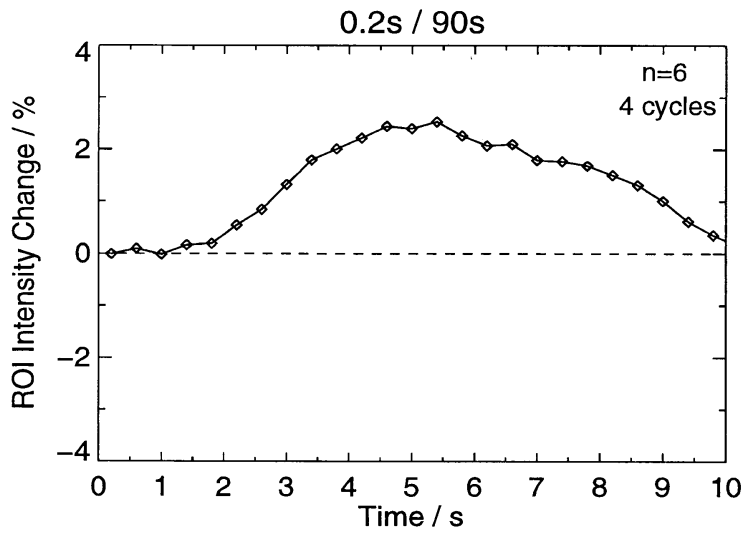

(C)

Fig. 3. Normalized time courses of BOLD MRI signal intensity changes $(0.4 \mathrm{~s}$ temporal resolution) for a protocol comprising 4 cycles of $0.2 \mathrm{~s}$ ofvisual activation (solid vertical lines) and $90 \mathrm{~s}$ of darkness. The curves represent mean values of all activated pixels relative to pre-stimulation baseline (horizontal broken line) and averaged across subjects $(n=6)$. A, real-time data of the entire protocol; $\mathrm{B}$, time-locked average of all four repetitive cycles of $90 \mathrm{~s}$ duration; $\mathrm{C}$, time-locked average of only the first $10 \mathrm{~s}$.

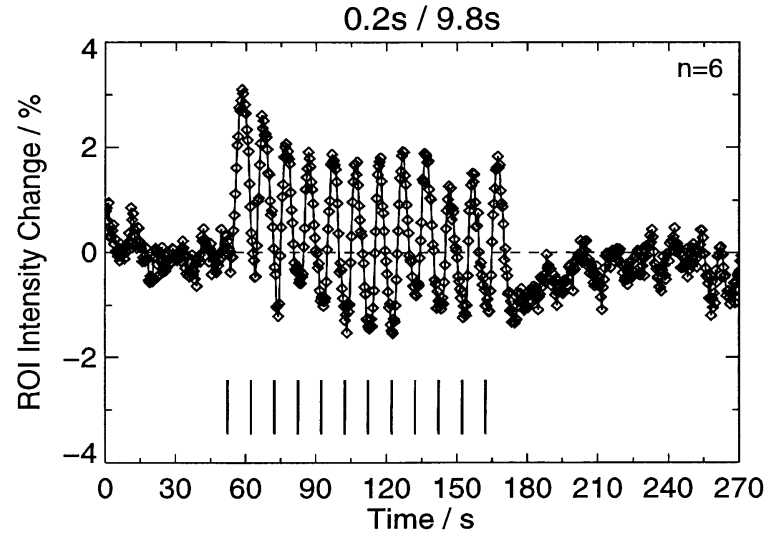

(A)

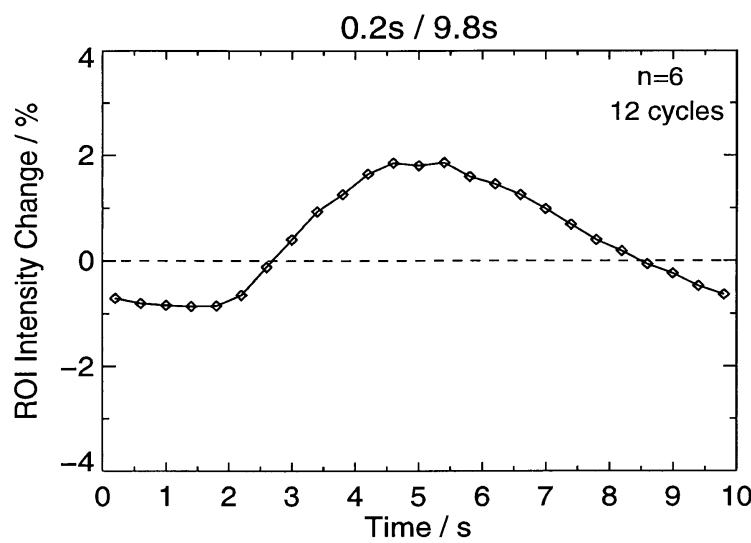

(B)

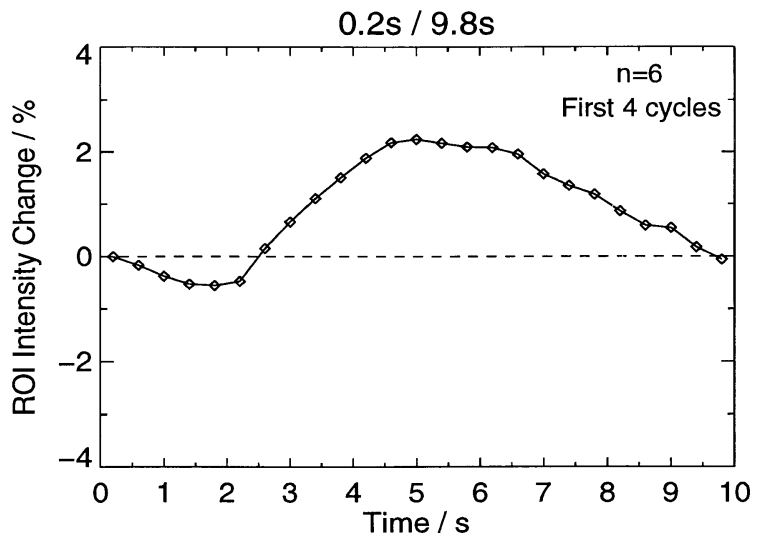

(C)

Fig. 4. Normalized time courses of BOLD MRI signal intensity changes $(0.4 \mathrm{~s}$ temporal resolution) for a protocol comprising 12 cycles of $0.2 \mathrm{~s}$ of visual activation (solid vertical lines) and $9.8 \mathrm{~s}$ of darkness. The curves represent mean values of all activated pixels relative to pre-stimulation baseline (horizontal broken line) and averaged across subjects $(n=6)$. A, real-time data of the entire protocol; $\mathrm{B}$, time-locked average of all 12 repetitive cycles of $10 \mathrm{~s}$ duration; $\mathrm{C}$, time-locked average of only the first four repetitive cycles. 
eraging of only the first 4 activation cycles covers the transition from pre-stimulation conditions to a new equilibrium (Fig. 4c) and gives rise to an artifactual signal decrease ("dip") of about $0.5 \%$ peaking at $1.5-2 \mathrm{~s}$ after onset of stimulation. This signal component is neither detected in the real-time data nor when using a $90 \mathrm{~s}$ control period (Fig. 3). It must be attributed to a "wraparound" effect from time-locked averaging caused by continuation of a preceding undershoot during the latency phase of a subsequent stimulus. This observation is in agreement with previous findings for a $1.6 \mathrm{~s} / 8.4 \mathrm{~s}$ protocol of rapidly repeated visual stimuli. ${ }^{15}$

\section{CONCLUDING REMARKS}

The key result of this study is that a subsecond visual stimulus leads to a similar oxygenation-sensitive MRI response as commonly observed for longer stimulus durations. The response profile includes 1) the lack of an "early response" or "initial dip"; 2) the well-known positive BOLD effect that most likely reflects a rapid increase in perfusion without a corresponding rise in oxygen consumption (or deoxyhemoglobin production); and 3) a subsequent undershoot phenomenon lasting for about 50-60 s after termination of the stimulus. Although the physical nature of this latter effect must be ascribed to an increased deoxyhemoglobin concentration, its physiologic mechanism has not been fully clarified. Hypotheses vary between an up-regulation of oxidative metabolism as also suggested for signal decreases during sustained visual activation ${ }^{18}$ and increases in cerebral blood volume. ${ }^{19}$

The present findings of extended MRI responses to very brief visual stimuli are of relevance for the interpretation of slow fluctuations in the temporal "noise" of MRI data sets that are obtained in the complete absence of functional challenge or task performance. This work provides experimental evidence that very brief cortical events or even spurious changes in activity during "resting" states may lead to physiologic consequences with time constants of several tens of seconds. It lends further support to studies by Biswal et al. ${ }^{20}$ who used corresponding low-frequency signal components to deduce spatial patterns of functionally connected neuronal activity.

Another consequence relates to baseline studies of the underlying physiology in magnetic resonance functional neuroimaging. To avoid complications from overlapping signal contributions, the control phases of pertinent experiments should be at least $60 \mathrm{~s}$. This ensures a "decoupling" of individual responses and minimizes influences from protocol timings on the interpretation of temporal MRI profiles. In fact, one may ask how "single" are "single trials" that are generated by event-related paradigms, if their MRI-detected physiologic history neces- sarily overlaps with that of multiple other trials. Clearly, linear relationships are only to be expected for a very limited range of stimulus features, stimulus durations, and inter-stimulus periods because the vascular responsiveness required for MRI detectability is limited by neuroanatomic constraints. In fact, preliminary assessments of pharmacologic interventions ${ }^{21,22}$ and cerebrovascular disease ${ }^{23}$ in conjunction with functional activation have indicated nonlinear interactions in human visual and motor cortex, respectively.

The more important question, therefore, is whether the physiologic history of cortical events alters the spatial activation pattern of the neuronal networks involved. Preliminary comparisons in this study (Fig. 2c,d) and in previous work (Fig. 2 in Fransson et al. ${ }^{15}$ ) support the view that modulations of the temporal response behavior do not affect the resulting activation maps and, therefore, will not hamper the future development of qualitative mapping of event-related activity changes.

Acknowledgments—We thank Dr. Martin Ingvar, Karolinska Institutet, Stockholm, for continuous interest and support.

\section{REFERENCES}

1. Savoy, R.L.; Bandettini, P.A.; O’Craven, K.M.; Kwong, K.K.; Davis, T.L.; Baker, J.R.; Weisskoff, R.M.; Rosen, B.R. Pushing the temporal resolution of fMRI: studies of very brief visual stimuli, onset variability and asynchrony, and stimulus-correlated changes in noise. In: Book of abstracts: Third Annual Meeting of the Society of Magnetic Resonance Imaging, New York, NY: ISMRM; 1995: p. 450 .

2. Blamire, A.M.; Ogawa, S.; Ugurbil, K.; Rothman, D.; McCarthy, G.; Ellermann, J.M.; Hyder, F.; Rattner, Z.; Shulman, R.G. Dynamic mapping of the human visual cortex by high-speed magnetic resonance imaging. Proc. Natl. Acad. Sci. USA 89:11069-11073; 1992.

3. McCarthy, G.; Luby, M.; Gore, J.C.; Goldman-Rakic, P. Functional magnetic resonance imaging in a visual oddball task. Neuroimage 3:548; 1996.

4. Buckner, R.L.; Bandettini, P.A.; O’Craven, K.M.; Savoy, R.L.; Petersen, S.E.; Raichle, M.E.; Rosen, B.R. Detection of cortical activation during averaged single trials of a cognitive task using functional magnetic resonance imaging. Proc. Natl. Acad. Sci. USA 93:14878-14883; 1996.

5. Dale, A.M.; Buckner, R.L. Selective averaging of rapidly presented individual trials using fMRI. Hum. Brain Map. 5:329-340; 1997.

6. Konishi, S.; Yoneyama, R.; Itagaki, H.; Uchida, I.; Nakajima, K.; Kato, H.; Okajima, K.; Koizumi, H.; Miyashita, Y. Transient brain activity used in magnetic resonance imaging to detect functional areas. Neuroreport 8:19-23; 1996.

7. Boynton, G.M.; Engel, S.A.; Glover, G.H.; Heeger, D.J. Linear systems analysis of functional magnetic resonance imaging in human V1. J. Neuroscience 16:4207-4221; 1996. 
8. Josephs, O.; Turner, R.; Friston K. Event-related fMRI. Hum. Brain Map. 5:243-248; 1997.

9. Friston K. Josephs, O.; Rees, G.; Turner R. Nonlinear event-related responses in fMRI. Magn. Reson. Med. 5:243-248; 1998.

10. Bandettini, P.A.; Kwong, K.K.; Davis, T.L.; Tootell, R.B.H; Wong, E.C.; Fox, P.T.; Belliveau, J.W.; Weisskoff, R.M.; Rosen, B.R. Characterization of cerebral blood oxygenation and flow changes during prolonged brain activation. Hum. Brain Map. 5:93-109; 1997.

11. Krüger, G.; Kleinschmidt, A.; Frahm J. Stimulus dependence of oxygenation-sensitive MRI responses to sustained visual activation. NMR Biomed. 11:75-79; 1998.

12. Chen, W.; Zhu, X.H.; Kato, T.; Andersen, P.; Ugurbil, K. Spatial and temporal differentiation of fMRI BOLD responses in primary visual cortex of human brain during sustained visual stimulation. Magn. Reson. Med. 39:520$527 ; 1998$

13. Hu, X.; Le, T.H.; Ugurbil, K. Evaluation of the early response in fMRI in individual subjects using short stimulus duration. Magn. Reson. Med. 37:877-884; 1997.

14. Janz, C.; Speck, O.; Hennig, J. Time-resolved measurements of brain activation after a short visual stimulus: New results on the physiological mechanisms of the cortical response. NMR in Biomed. 10:222-229; 1997.

15. Fransson, P.; Krüger, G.; Merboldt, K.D.; Frahm, J. Temporal characteristics of oxygenation-sensitive MRI responses to visual activation in humans. Magn. Reson. Med. 39:912-919; 1998.

16. Bandettini, P.A.; Jesmanowicz, A.; Wong, E.C.; Hyde, J.S. Processing strategies for time-course data sets in functional MRI of the human brain. Magn. Reson. Med. 30:161-173; 1993.
17. Kleinschmidt, A.; Requardt, M.; Merboldt, K.D.; Frahm, J. On the use of temporal correlation coefficients for magnetic resonance mapping of functional brain activation. Individualized thresholds and spatial response delineation. Intern. J. Imag. Sys. Technol. 6:238-244; 1995.

18. Krüger, G.; Kleinschmidt, A.; Frahm J. Dynamic MRI sensitized to cerebral blood oxygenation and flow during sustained activation of human visual cortex. Magn. Reson. Med. 35:797-800; 1996.

19. Buxton, R.B.; Wong, E.C.; Frank, L.R. A biomechanical interpretation of the BOLD signal time course: The balloon model. In: Book of abstracts: Fifth Annual Meeting of the International Society of Magnetic resonance Imaging, Vancouver, Canada: ISMRM, 1997: p. 743.

20. Biswal, B.; Yetkin, F.Z.; Haughton, V.M.; Hyde, J.S. Functional connectivity in the motor cortex of resting human brain using echo-planar MRI. Magn. Reson. Med. 34:537-541; 1995.

21. Bruhn, H.; Kleinschmidt, A.; Boecker, H.; Merboldt, K.D.; Hänicke, W.; Frahm J. The effect of acetazolamide on regional cerebral blood oxygenation at rest and under stimulation as assessed by MRI. J. Cereb. Blood Flow Metab. 14:742-748; 1994.

22. Kleinschmidt, A.; Bruhn, H.; Steinmetz, H.; Frahm, J. Pharmacologic manipulation of vasomotor tone studied by magnetic resonance imaging of cerebral blood oxygenation. J. Cerebr. Blood Flow Metab. 15: S527; 1995.

23. Kleinschmidt, A.; Steinmetz, H.; Sitzer, M.; Merboldt, K.D.; Frahm, J. Magnetic resonance imaging of regional cerebral blood oxygenation changes under acetazolamide in carotid occlusive disease. Stroke 26: 106-110; 1995. 